DE LA COMPETENCIA COMUNICATIVA A LA COMPETENCIA

\title{
INTERCULTURAL: UNA PROPUESTA TEÓRICA Y DIDÁCTICA PARA LA EDUCACIÓN INTERCULTURAL EN LATINOAMÉRICA
}

\author{
Delia María Fajardo Salinas \\ demafasa@yahoo.com \\ Universidad Pedagógica Nacional Francisco Morazán \\ Honduras
}

\section{RESUMEN}

En este artículo se revisa la evolución del concepto de competencia comunicativa desde que fuera propuesto por Dell Hymes, pasando por los principales modelos de análisis de dicho constructo, con un énfasis en la relevancia que fue cobrando la formulación de ciertas capacidades y actitudes para afrontar la relación con la diferencia cultural, en el marco de la enseñanza de lenguas, todo ello como fundamento para elaborar una propuesta teórica del concepto de competencia intercultural factible de convertirse en el núcleo curricular de una propuesta didáctica para programas de educación intercultural en Latinoamérica. Entre los modelos de análisis considerados destacamos el de Cantero (2009), por la novedad de su aporte con un enfoque apoyado en la teoría de la complejidad para explicar las relaciones entre los elementos que integran la competencia comunicativa y, más importante aún, el potencial descriptivo y normativo de su modelo de análisis para la enseñanza de lenguas.

Palabras clave: competencia comunicativa, competencia intercultural, complejidad, educación intercultural, canon formativo intercultural. 


\section{ABSTRACT}

This paper reviews the evolution of the concept of communicative competence proposed by Dell Hymes, including the main models for the analysis of this construct with an emphasis on the relevance acquired by the formulation of certain capabilities and attitudes to face the relationship with cultural difference within the framework of language teaching. This is the foundation for the development of a theoretical proposal about the concept of intercultural competence that may feasibly become the curricular core of a didactic proposal for intercultural education programs in Latin America. Cantero's model (2009) is noted among the most outstanding analysis models because of the novelty of his contribution with an approach that uses complexity theory to explain both the relationships among the elements integrating communicative competence and even more importantly the descriptive and normative potential of his analysis model for language teaching.

Key words: communicative competence, intercultural competence, complexity, intercultural education, intercultural education model. 
Desde que Noam Chomsky formulara en 1965 su diferenciación entre competencia y actuación para referirse al conocimiento y uso de la lengua, respectivamente, el término competencia ha ido revelando su potencial epistemológico, sobre todo a partir de su alianza con el rasgo comunicativa como producto de las revisiones y críticas posteriores a la teoría chomskiana. Es decir, la categoría competencia comunicativa proviene de un proceso de crítica que dio lugar al desarrollo de dos líneas de investigación: la propia de la lingüística generativista, y otra que se interesó en los procesos y ámbitos reales de actuación lingüística de los hablantes, en lugar de los ámbitos descontextualizados y artificiales de un laboratorio lingüístico.

El pilar fundacional de esta tradición alternativa a la lingüística generativista es la teoría de los actos de habla, iniciada por John Austin y desarrollada por John Searle, ambos británicos. Pero el hallazgo del concepto competencia comunicativa se debe a Dell Hymes, sociolingüísta y antropólogo de origen estadounidense, interesado en el estudio de la relación entre las creaciones literarias — tradición oral, mitos, narraciones, poesías-, y formas de pensamiento. Con sus críticas a los conceptos chomskianos en 1966 buscó demostrar que saber una lengua es algo más que saber un vocabulario y un sistema gramatical. Es necesario poseer otros conocimientos no lingüísticos aunque siempre ligados a un tipo de codificación verbal o no verbal: convenciones de uso de acuerdo con factores sociales y creencias, es decir, de acuerdo con la cultura, en el amplio sentido de este término. Hymes nos hizo ver que dejar fuera del análisis tales elementos no permite visualizar el fenómeno comunicativo en toda su integridad y complejidad.

Las contribuciones posteriores a Hymes fortalecieron el cambio de enfoque que modificó la manera de entender qué es el lenguaje y la comunicación, entre las principales: Selinker (1972), con el concepto de interlengua; Grice (1975), con el principio de cooperación; Krashen (1977), con la diferenciación entre adquisición y aprendizaje; Halliday (1978), con un modelo de funciones de la comunicación, y Widdowson (1978), con un modelo comunicativo de enseñanza de lenguas (en Mendoza y Cantero 2003: 49). La importancia de estas y otras aportaciones radica en que fueron configurando los fundamentos teóricos del enfoque comunicativo, por el 
cual se dejó de concebir la lengua como conocimiento gramatical para hablar un idioma, y se pasó a entender como un conocimiento declarativo y operativo necesario para la comunicación.

El concepto de competencia comunicativa continúa siendo objeto de revisiones, cambios y ampliaciones, que son consecuentes con las ideas originales. En los ya casi cuarenta años trascurridos, percibimos que existe un acuerdo tácito en que la competencia comunicativa es el «...conjunto de conocimientos y habilidades que un hablante debe haber adquirido a lo largo de su vida para enfrentarse a una situación comunicativa concreta, y a la capacidad de afrontar con éxito los múltiples condicionantes de la comunicación» (ibíd: 49).

Sin embargo, no pasa igual con respecto a un modelo que explique el sistema de funcionamiento de esos conocimientos y habilidades. En el esfuerzo por lograr una mejor explicación que la anterior, con la que diferenciar con claridad y exactitud los conocimientos y habilidades implicados y los mecanismos de interacción cuando alguien se comunica, cada nueva propuesta evidencia lo mucho que se ha avanzado desde las categorías provisionales - pero fundacionales - del modelo original de Hymes.

Una de las propuestas más relevantes por su decisiva influencia posterior es la de Jan A. van Ek y J. L. M. Trim (1991). Dentro de sus proyectos para el desarrollo de las lenguas modernas europeas, el Consejo de Europa asignó a estos lingüistas británicos el diseño de una programación curricular para el aprendizaje del inglés por adultos que dieron en llamar Nivel Umbral; en su metodología de trabajo, se basaron en el análisis de las necesidades del grupo meta para la definición de los objetivos de aprendizaje, y consideraron que el dominio del código lingüístico no es un fin en sí mismo, sino un instrumento para la actuación comunicativa. Este trabajo marcó un nuevo camino que se alejó de las metodologías estructuralistas de base conductista, y colaboró en que se avanzara más decisivamente hacia la instauración del enfoque comunicativo en la enseñanza de lenguas. 
Un ejemplo de las investigaciones más destacadas que surgieron en esta nueva tradición pedagógica es el de Canale \& Swain (1980), quizá por el mérito de organizar en unas pocas categorías esa larga lista de aprendizajes, un tanto dispersa, como parte de la competencia comunicativa ideal que debía alcanzar un estudiante de lengua extranjera en el programa del Nivel Umbral. Además buscaban la facilidad de traducción pedagógica de las formulaciones teóricas para su comprensión y traslado operativo a las aulas. Estos autores propusieron como componentes cuatro áreas: lingüística, discursiva, estratégica y sociolingüística. Sin embargo, en ellos todavía no hay una consideración explícita de la problemática de las sociedades multiculturales y del tipo de formación que a través del aprendizaje de un idioma se puede efectuar para afrontarla.

Esto cambiará en 1988 con la publicación de Objectives for Foreign Language Learning, documento en el cual van Ek presenta un marco teórico referencial que describe integralmente los elementos que se consideran básicos para que un hablante alcance una comunicación eficaz en el idioma inglés; entre los cuales incluye, además de las ya definidas por Canale \& Swain, una subcompetencia sociocultural y otra social - que implica específicamente repercusiones en el desarrollo de la personalidad, en términos de desarrollo cognitivo y emocional- (van Ek 1993: 34-36). Este marco teórico referencial ofrece un análisis de la «habilidad comunicativa» a partir del cual se pueden establecer los objetivos específicos de aprendizaje en la enseñanza de la lengua meta.

Por el interés de este artículo en llegar a una definición de la competencia intercultural, cabe ampliar sobre la definición de la subcompetencia sociocultural; de acuerdo con van Ek, el desarrollo de este componente conduce al hablante a ampliar su horizonte cultural y descubrir la validez de otras formas de categorizar la realidad y la experiencia, y otros modos de manejar las relaciones humanas en situaciones diversas de la vida. Por ende, se supone que este conocimiento evitará el manejo de estereotipos y que se desarrollen actitudes positivas hacia la diferencia cultural así como la habilidad de estar atentos ante las connotaciones culturales ligadas a ciertos usos lingüísticos, y de aprender las 
expresiones convencionales para rituales sociales de la cotidianidad, entre otros aspectos que de hecho poseen una relevancia educativa general y de lo cual se deriva su crucial importancia (ibíd: 59).

Para conducir al desarrollo de la competencia sociocultural, van Ek enfatiza la necesidad de complementar información con reflexión, es decir, proveer datos sobre la o las otras culturas y también realizar actividades que involucren de manera personal al estudiante, en sus actitudes, opiniones, sistema de valores y gestión de las emociones. Específicamente, recomienda la discusión de temas sensibles desde ambas perspectivas culturales, de los estudiantes y de los hablantes nativos. Para el caso particular de los inmigrantes en Europa, van Ek advierte que la competencia sociocultural seguramente ocupará la mayor parte del tiempo de aprendizaje para favorecer el proceso de adaptación, ya que desarrollar dicha competencia es casi una condición clave para sobrevivir (ibíd: 62). Y en el caso de la educación en Latinoamérica creemos que tendría que ocupar un espacio relevante no tanto en el aprendizaje de lenguas de los indígenas o afrodescendientes como de los mestizos, de la población urbana o de las clases sociales altas, ya que es en estos grupos sociales donde se alimentan esos estereotipos y actitudes negativas de rechazo hacia el otro que es diferente tanto étnica como socialmente. Como se verá adelante en la definición de conciencia intercultural en la propuesta educativa europea, lo que se busca es superar el etnocentrismo, como punto de partida para que sean posibles cambios estructurales en las formas de gobierno y definición de políticas sociales orientadas al bien común.

También consideramos un aporte importante la relevancia que van Ek asigna a los textos literarios para el desarrollo de la competencia sociocultural. Básicamente nos recuerda esa cualidad polifónica de la cual nos habla M. Bajtin, es decir, el hecho de que cada uno de los personajes puede proyectar una visión de mundo, haciendo del texto literario un tamiz de voces, de perspectivas, todas puestas en relación según la trama narrativa, que se pueden apreciar desde una relación a distancia o reconociendo afinidad con las motivaciones y experiencias de los personajes. De esta manera, el lector entra en contacto íntimo y personal con los sentidos más significativos de la cultura allí escrita, que definen la forma de ser de los hablantes nativos: «...through the judicious 
choice of texts [a learner] will be enable to share some of the formative experiences of native speakers of the foreign language, thus improving his quality as a communication partner» (ibíd).

Precisamente, en este artículo queremos argumentar que dicha utilidad de las narraciones para la formación de la competencia sociocultural es factible de convertirse en la estrategia nuclear de una propuesta didáctica, orientada al aprendizaje y desarrollo de la competencia intercultural en la educación básica de toda la población de una nación, con el propósito de superar el etnocentrismo y demás actitudes negativas hacia la diversidad cultural, objetivo global subyacente de estas iniciativas teóricas y pedagógicas, como se verá más adelante.

Continuando con la reseña sobre la génesis y evolución del concepto de competencia comunicativa, llegamos al momento en que el Consejo de Europa - luego de no cesar el apoyo a proyectos de investigación en la enseñanza de segundas lenguas, de la exitosa recepción de los trabajos de van Ek y de las adaptaciones que se reprodujeron en otros países para las otras lenguas europeas-, logró consolidar una plataforma sólida para elaborar una propuesta curricular estándar — pero flexible - para la enseñanza de lenguas en el continente. Surge así el Common European Framework of Reference for Languages (2001) [en adelante MRE]. Este documento marca de forma definitiva la institucionalización del enfoque comunicativo para la enseñanza de lenguas, que lo llama «enfoque basado en la acción», inicia con una visión muy amplia del uso y aprendizaje de lenguas, en la cual los actos de habla, aunque se dan en actividades de lengua, forman parte de un contexto de acción más amplio donde se «considera a los usuarios y alumnos que aprenden una lengua principalmente como agentes sociales, es decir, como miembros de una sociedad que tiene tareas [no sólo relacionadas con la lengua] que llevar a cabo en una serie determinada de circunstancias, en un entorno específico y dentro de un campo de acción concreto» (Consejo de Europa 2001: 24). Por lo anterior, se establece una diferenciación de tipos de competencias que excede el campo lingüístico: «El uso de la lengua [incluye el aprendizaje] comprende acciones llevadas a cabo por personas que, como individuos y como agentes sociales, desarrollan una serie de competencias, tanto generales como competencias comunicativas, en 
particular» (ibíd). Y además, en lugar de manejar como objetivo meta el desarrollo de cuatro destrezas — comprensión oral, comprensión escrita, expresión oral y expresión escrita-, en el nuevo paradigma lo deseable es alcanzar el dominio de la lengua meta en cuatro áreas de acción comunicativa: producción y recepción — que abarcan las destrezas anteriores-, y además interacción y mediación.

Podríamos resumir que MRE presenta unas características muy ventajosas para el trabajo pedagógico en aspectos donde el enfoque por destrezas se quedaba limitado, ya que:

- $\quad$ supera el reduccionismo anterior, ya que no promueve únicamente el aprendizaje de un código lingüístico sino la capacidad de comunicarse, considerando lo polifacético de esta actuación;

- $\quad$ enriquece y da cabida a las actividades comunicativas que quedaban fuera —interacción y mediación-;

- $\quad$ su definición genérica de competencias permite su aplicabilidad en términos de cualquier código;

- $\quad$ introduce con especial relevancia la capacidad de mediación en el concepto de competencia comunicativa.

Nos interesa destacar la novedad de este último aspecto, ya que responde a los objetivos políticos y educativos generales del Consejo de Europa, en particular: «to promote the personal development of the individual $[\ldots]$ as an active agent $[\ldots]$ and a well-informed, positive attitude towards other peoples and their cultures, free from prejudice, intolerance and xenophobia» (Trim 1997: 5-6). Es decir, hay un reconocimiento de la necesidad de tratar de forma explícita en el currículo escolar el desarrollo de una competencia que prepare a las personas para saber afrontar y conducirse ante las problemáticas que se derivan de la realidad multilingüe y multicultural que caracteriza a la geografía europea, y de hecho a la mayoría de sociedades actualmente, como condición necesaria para facilitar la integración social en función del bien común y desarrollo humano, y por ende del desarrollo de cada país y de la Unión Europea en su conjunto. Por lo tanto, el objetivo no es el dominio de «un idioma», sino que «to build up mutual understanding and acceptance of cultural and linguistic diversity in a 
multilingual and multicultural Europe» (ibíd). En tal sentido, la importancia de la competencia mediadora es enunciada con acierto por Cantero y De Arriba: «...no es una mera novedad metodológica, sino una actividad esencial de supervivencia en las relaciones humanas que se desarrollan en contextos plurilingües» (2004: 2).

Sin duda, la reforma educativa que MRE ha impulsado, o de la cual es parte, está motivada fuertemente por esta nueva mirada a sus sociedades, y representa por ello una declaración de aspiraciones en torno al tipo de desarrollo que se desea para los ciudadanos del continente, así como de desarrollo sociopolítico para cada Estado de la Unión. De ahí que se ejemplifique constantemente en las evidencias de logro de las competencias generales y específicas cómo se puede ejercer y potenciar esta capacidad de mediación - lo entendemos como interés en promover un comportamiento intercultural-. En otras palabras, el MRE está repleto de la descripción de las conductas interculturales deseables que se buscan desarrollar en los ciudadanos, ya sea como competencia general o como competencia lingüística, o como competencia plurilingüe y pluricultural. Esta fuerte y decidida promoción de la interculturalidad está concentrada en los capítulos 5 y 8 de MRE. Dada su amplitud, en este espacio solamente retomaríamos algunos fragmentos, porque nos servirán para la definición que aquí se quiere proponer de competencia intercultural en función del contexto social y educativo latinoamericano, y porque es el antecedente inmediato del siguiente planteamiento teórico sobre la competencia comunicativa que pasaremos a resumir después, el de Cantero (2009) y su perspectiva desde el pensamiento complejo.

En primer lugar, queremos destacar cómo se define la conciencia intercultural en MRE: como el producto del «conocimiento, la percepción y la comprensión de la relación entre el "mundo de origen" y el "mundo de la comunidad objeto de estudio" (similitudes y diferencias distintivas)». Implica, además: «la conciencia de una serie de culturas más amplia de la que conlleva la lengua materna y la segunda lengua, lo cual contribuye a ubicar ambas en su contexto», esto supone «una toma de conciencia del modo en que aparece la comunidad desde la perspectiva de los demás, a menudo en forma de estereotipos nacionales» (Consejo de Europa 2001: 101) [desde este punto, en las citas de MRE se indicará solamente el número de página]. 
Con base en lo anterior, la conciencia intercultural tiene como correlato su expresión en ciertas destrezas y habilidades de la competencia del saber hacer:

- «La capacidad de relacionar entre sí la cultura de origen y la cultura extranjera.

- La sensibilidad cultural y la capacidad de identificar y utilizar una variedad de estrategias para establecer contacto con personas de otras culturas.

- La capacidad de cumplir el papel de intermediario cultural entre la cultura propia y la cultura extranjera y de abordar con eficacia los malentendidos interculturales y las situaciones conflictivas.

- La capacidad de superar relaciones estereotipadas» (102).

En la competencia del saber ser, el eje trasversal de la interculturalidad cobra mayor definición como pieza nuclear, ya que dicha competencia se relaciona directamente con la identidad cultural de cada persona, entonces, es el punto más sensible para las percepciones y las relaciones con la otredad. De ahí que se reflexione en cómo la actividad comunicativa está determinada por características individuales: actitudes, motivaciones, valores, creencias, estilo cognitivo, todo lo que configura la identidad de la persona. Lo de mayor interés didáctico es las actitudes a formar:

- «Apertura hacia nuevas experiencias, otras personas, ideas, pueblos, sociedades y culturas, y el interés que muestran hacia ello;

- voluntad de relativizar la propia perspectiva cultural y el propio sistema de valores culturales;

- voluntad y capacidad de distanciarse de las actitudes convencionales en cuanto a la diferencia cultural [racismo, xenofobia...]» (103).

Es decir, se describe un perfil de persona totalmente trasfigurado por la filosofía de la interculturalidad; ciertamente el Consejo de Europa valora que el «desarrollo de una personalidad intercultural sea considerado una meta educativa importante en sí mismo por muchos» (104). En otras palabras, aprender sobre los riesgos que implican los malentendidos culturales — por un error de audición, por ejemplo- puede ser el objetivo principal que se justifica por sí mismo en un proceso de aprendizaje de segunda lengua. 
La importancia dada a la formación de una personalidad intercultural a través de aprendizajes lingüísticos recibe otro apoyo con la concepción de las competencias pluricultural y plurilingüe: «un individuo no tiene un repertorio de competencias diferenciadas y separadas para comunicarse dependiendo de las lenguas que conoce, sino una competencia plurilingüe y pluricultural que incluye el conjunto de esas lenguas» (167). Por ello, MRE justifica la introducción de una lengua extranjera, LE1, a nivel primario, como mecanismo para potenciar el desarrollo de las competencias generales del individuo, entre las cuales se menciona: «el descubrimiento o el reconocimiento [...] de la pluralidad de lenguas y culturas, la preparación para alejarse del etnocentrismo, la relativización pero también la confirmación de la identidad lingüística y cultural del alumno» (170-171).

Para concluir aquí, valoramos la mirada holística que el Consejo de Europa asume para desarrollar el concepto de competencia comunicativa en un marco teórico sumamente amplio, de manera que incluye una teoría del desarrollo cognitivo, de la acción humana e incluso del desarrollo social general. Por otra parte, apreciamos como un rasgo discursivo clave de MRE la presencia de la filosofía intercultural como un aire que se respira en todo el documento, es decir, aunque no encontramos una definición explícita o aislada de competencia intercultural; un tratamiento como competencia autónoma, en lugar de ello, es destreza, es conciencia, es actitud. Nos parece muy acertado que la interculturalidad se desglose conceptualmente en todas las competencias generales y específicas, con lo cual se reconoce como una dimensión que debe permear integralmente toda la modelación sicológica de las personas.

Por todo ello, MRE nos parece un punto culminante, aunque no final, en la discusión e investigación sobre la enseñanza de lenguas, ya que si se toma como modelo a ser replicado en otros contextos sociales es posible chocar con estructuras y políticas de gobierno contrarias al espíritu de este programa educativo en el área de lenguas. Es decir, se debe tener bien claro que el plano desiderativo de un currículo debe tener su correlato con unas condiciones institucionales que permitan su implementación, como aparentemente ocurre en Europa desde estas propuestas oficiales. 
En el caso particular de Latinoamérica, en muchos países hay todavía mucho trecho por recorrer para que las clases gobernantes asuman una posición visionaria del desarrollo de la nación, bajo el entendido que la diversidad cultural y lingüística no es una amenaza sino por el contrario un potencial a explotar, y que no tiene por qué afectar la construcción de la comunidad política, sustentada en un contrato social entre sus miembros para alcanzar prosperidad y calidad de vida para todos. En otras palabras, falta entender que la unidad política no tiene por qué exigir homogeneidad cultural (Fábregas 2011). En cambio, la inequidad en la distribución de la riqueza, las injusticias sociales y otros errores de gobernabilidad, sí socaban directamente la ansiada armonía sociopolítica. Sin duda esto es algo a tomar en cuenta para viabilizar la implementación de cualquier programa educativo alternativo que atienda necesidades de grupos marginados socialmente, para así enfocar los esfuerzos en un cambio de mentalidad en las clases o grupos sociales que se sostienen por y reproducen este tipo de creencias políticas tradicionales que retardan el desarrollo humano y social de la nación, como es la intención de la propuesta didáctica que presentaremos más adelante.

Hasta aquí hemos hecho referencia a unos modelos de análisis muy influyentes sobre competencia comunicativa y su tratamiento de la noción interculturalidad. Para finalizar, haremos referencia a la propuesta más reciente que conocemos: el modelo de Cantero (2009). Consideramos que este autor aporta una nueva mirada al fenómeno y representa una verdadera innovación en los estudios sobre la competencia comunicativa, por su acertada explicación de la compleja dinámica en que se relacionan integralmente los componentes de dicha competencia cuando se activan según las actividades que los hablantes realizan, esfuerzo teórico que en el mismo MRE se asume casi como imposible: «...una completa representación, en forma de diagrama, del grado de multidimensionalidad abarcado [para definir la competencia comunicativa] sería, en realidad, muy complicada, si no imposible» (Consejo de Europa 2001: 16). Como se verá, Cantero trasfiere y abrevia en unos pocos esquemas lo que en el Nivel Umbral y en MRE se desglosa ampliamente para entender el aprendizaje de lenguas contemplado desde la perspectiva del desarrollo humano y en función de objetivos educativos y sociales globales. 
En su análisis, Cantero retoma de MRE la diferenciación entre competencias generales y competencias comunicativas específicas. No obstante en el primer grupo reúne las cuatro categorías ya propuestas por Canale \& Saiwn (1980):

- competencia lingüística

- competencia discursiva

- competencia estratégica

- competencia cultural

Las definiciones de estas subcompetencias no difieren en su esencia de propuestas anteriores, aunque se añaden importantes matices. Por ejemplo: ver en el comportamiento de la competencia estratégica la manifestación de la «zona de desarrollo próximo», puesto que consiste en la capacidad de «relacionar todas las demás competencias para gestionar las diversas situaciones comunicativas a que se enfrenta el hablante, mediante los recursos que ha ido generando y elaborando a lo largo de su vida, [...] adaptarse a nuevos contextos e interlocutores, así como su capacidad de aprender/adquirir nuevos códigos» (Cantero 2009). En otras palabras, en activación de esta subcompetencia el hablante demuestra tanto los aprendizajes ya logrados en el dominio del código, de las adecuaciones pragmáticas, o de las convenciones culturales, y además puede demostrar lo que es capaz de hacer, actuando creativamente, fuera de las situaciones regulares y automáticas de la comunicación en su vida cotidiana ante situaciones conflictivas o ámbitos culturales nuevos. Por tanto, es la capacidad nuclear que moviliza el resto de recursos cognitivos, y su desarrollo sería el principal objetivo dentro de los parámetros de la pedagogía constructivista.

Por otra parte, Cantero opta por el término cultura para referirse a lo que otros han llamado sociolingüístico, sociocultural o social, y define esta competencia como:

\footnotetext{
la capacidad de manejar los saberes implicados en la comunicación, desde los significados de las palabras y sus connotaciones hasta las implicaturas conversacionales y las intenciones reflejadas en el discurso [los contenidos ilocutivo y perlocutivo]. Todo el mundo nocional comprometido con el discurso, incluido el modelo del mundo del hablante, así como los contenidos culturales específicos del grupo a que pertenece (ibíd).
} 
En el segundo grupo coloca las actividades comunicativas específicas:

- competencia productiva

- competencia perceptiva

- competencia interactiva

- competencia mediadora

En este caso, la capacidad nuclear y más importante es la competencia interactiva, por varias razones que el autor argumenta: sin interacción no surge la comunicación, con la interacción es que se adquiere la lengua, mediante la interacción se gestiona nuestra vida cotidiana y relaciones personales... en fin, la interacción posibilita nuestra misma existencia.

Hasta este punto, la propuesta de Cantero no representaría un avance significativo en relación con sus antecedentes, pero creemos que su análisis del funcionamiento sistémico e integral de estas dimensiones y recursos que conforman la competencia comunicativa es su aporte más significativo. Desde un inicio, este autor enfatiza que todas las subcompetencias contempladas no operan en paralelo, porque la competencia comunicativa «constituye una única unidad operativa, cuyos componentes están perfectamente integrados [...] y cuya complejidad conviene comprender» (ibíd).

Dicha complejidad se manifiesta en que cada competencia general se compone a su vez de todas las competencias específicas. Esto significa que la competencia lingüística, la discursiva, la cultural y la estratégica, generan distintas manifestaciones de competencia productiva, perceptiva, mediadora e interactiva. Por ejemplo, una persona puede tener muy desarrollada su competencia mediadora en el campo de su competencia lingüística, pero no así en la cultural; en consecuencia, aunque pueda traducir palabras de un idioma a otro, no será capaz de interpretar y gestionar los significados culturales entre hablantes, peor aun: su interacción será torpe porque no tendrá suficientes recursos para actuar estratégicamente y negociar entre sus interlocutores, con lo cual su comunicación puede fracasar. 
Este modelo sobre el sistema complejo de funcionamiento en la competencia comunicativa presenta como característica que puede tener un uso normativo: indicar el desarrollo ideal de la competencia comunicativa en todos para sustentar una programación curricular, así como un uso descriptivo, con los que detectar las carencias o desarrollo desigual de las subcompetencias en un hablante, es decir, para describir su «interlengua» en términos de competencia comunicativa. Cantero plantea una serie de ejemplos de casos típicos en el aprendizaje de lenguas para demostrar cómo su modelo de análisis efectivamente ofrece una explicación razonable de las causas de ciertas deficiencias en la actuación comunicativa de las personas. Por ejemplo, en el caso de «alumnos que identifican las palabras en un listening pero no pueden seguir una conversación, han desarrollado una competencia perceptiva en su competencia lingüística pero no en su competencia discursiva» (ibíd). Esta forma de explicar la interlengua de un estudiante, ya sea como diagnóstico o evaluación al final de un curso, posee una novedad que otros modelos no ofrecían con tal sencillez, y consideramos que viene a ser otro mérito de esta propuesta. En MRE, al menos, ya se advierte que no es posible medir linealmente el dominio progresivo de una lengua (Consejo de Europa 2001: 17-18).

Volviendo al análisis de Cantero, y como habíamos mencionado, el autor ofrece un esquema ilustrativo: una imagen compleja que denomina «triángulo múltiple», y que representa cómo es la competencia comunicativa en un código (ver figura 1): 
Figura 1. Triángulo múltiple de subcompetencias

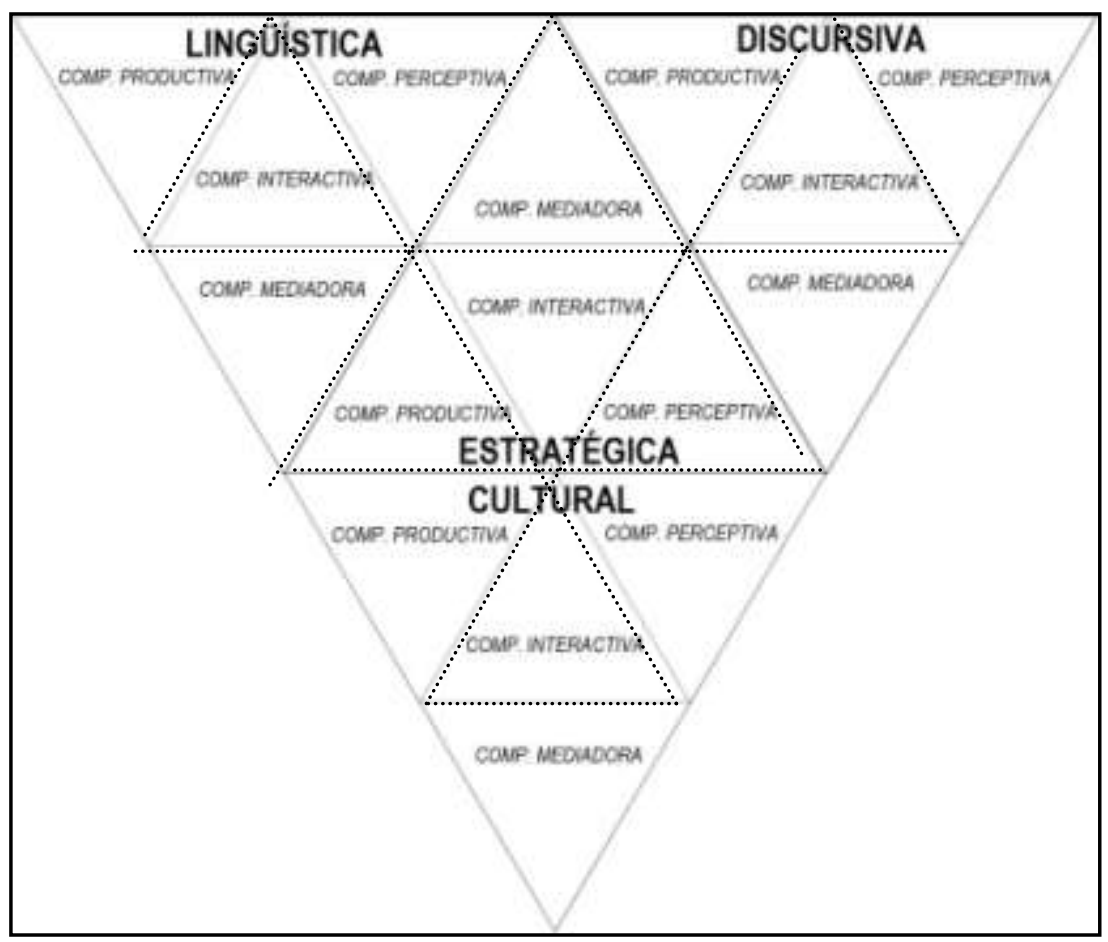

Fuente: Cantero, Francisco José, 2009, «Complejidad y competencia comunicativa», en Congreso Horizontes de Lingüística Aplicada, Brasilia, Brasil.

Hemos añadido líneas punteadas para denotar el énfasis del autor en que «el desarrollo de cada [compartimento] afecta y modifica el desarrollo de todos los demás, continuadamente» (ibíd). Es decir, no son fronteras que separan sino vasos comunicantes que permiten la colaboración entre las distintas capacidades para su funcionamiento y desarrollo evolutivo.

Puesto que tal esquema grafica cómo es la competencia comunicativa en un solo código —un hablante monolingüe ficticio—- el autor elabora un siguiente esquema más fiel a la realidad, es decir, partiendo del hecho de que un hablante maneja diversos códigos de forma alternativa o simultánea (Mendoza \& Cantero 2003; Cantero 2004, 2009) y que, por tanto, en cada uno manifiesta cierto nivel de desarrollo de competencia comunicativa. 
Los ejemplos que el autor presenta, con respecto a la diversidad de códigos y sus relaciones, justifican su postura: «Suponer una única competencia comunicativa que sirve para todo sería caer en un reduccionismo inútil. Más adecuado es intentar comprender la complejidad de nuestra competencia comunicativa atendiendo también a la diversidad de códigos que empleamos espontánea y masivamente» (2009).

Por ello, la competencia comunicativa real de un hablante puede representarse como una estrella de varios triángulos múltiples, tantos como códigos maneje:

Figura 2. La estrella comunicativa

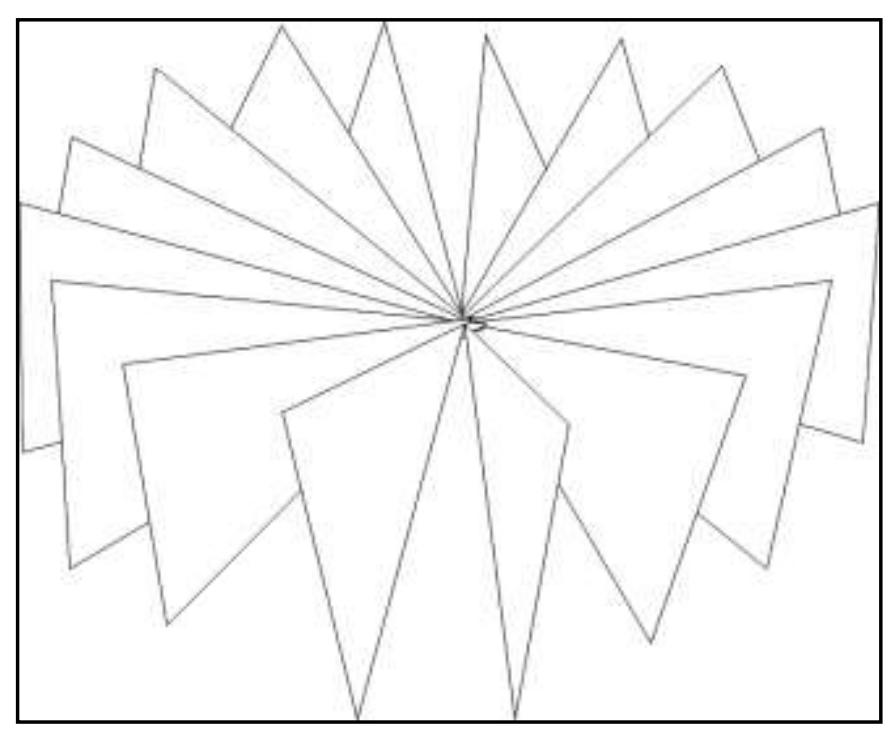

Cantero, Francisco José, 2009, «Complejidad y competencia comunicativa», en Congreso Horizontes de Lingüística Aplicada, Brasilia, Brasil.

Esta es una estrella «abierta», porque la competencia comunicativa se encuentra en formación permanente: siempre es posible aprender nuevos códigos y mejorar el desarrollo de las subcompetencias en cada uno y globalmente: «La estrella comunicativa sería como un libro de arena, con un número indefinido de hojas, de códigos que el hablante va elaborando a lo largo de su vida, número que puede variar y crecer, también indefinidamente» (ibíd). Los lindes entre las hojas de la estrella son permeables, aunque cada hoja tenga un desarrollo por separado — L1 estará más desarrollada que L2, el dominio de la expresión oral puede ser mayor que el de la expresión escrita, el manejo del nivel paralingüístico puede ser menor que el del 
lingüístico- Entonces, con el desarrollo de la competencia estratégica —entiéndase: de la «zona de desarrollo próximo»-, motor de la competencia comunicativa, estaremos estimulando el desarrollo del resto de componentes y, por ende, de toda la estrella.

En este punto nos acercamos a la parte del análisis que más nos interesa: la incorporación de la competencia cultural. Cantero reflexiona sobre el valor de esta competencia en todo el esquema: en primer lugar, es la base de la identidad del hablante; en segundo lugar, es común en todos los triángulos de la estrella comunicativa, o sea, a todos los códigos —o a la mayoría-, puesto que usar uno u otro no implica salir del propio modelo de mundo y asumir otro radicalmente; finalmente, aunque su existencia es estable, no significa que no pueda cambiar para convertirse en una competencia intercultural, en el sentido de volverse receptiva y plástica ante otros modelos de mundo, a contextos y hablantes nuevos. En palabras de Cantero, elaborar «un modelo del mundo que integre y acepte la diversidad, y un patrón de significados semánticos, pautas sociales y actitudes, que entienda y asuma la diferencia» (ibíd).

Vemos que la competencia intercultural se inserta en la parte más nuclear de la persona: su identidad sicológica individual, su modelo de mundo, sus actitudes, su carácter. El término inter-cultura, en lugar de pluri-cultura, que se utiliza en MRE, permite denotar que este conocimiento de varias culturas se mezcla de tal forma que sustenta la identidad de la persona con base en los mínimos comunes, su modelo de mundo original o primario a partir de lo mínimo común con otros modelos de mundo, lo cual genera un proceso de cambio: el pensamiento puede desplazarse o hacer acopio de entre todos los significados culturales que ha aprendido no solo para comunicarse con éxito, sino para configurar constantemente una nueva identidad: adoptar unos significados, reciclar otros, marginar algunos más, etc., de manera que la mente arma su propia conjugación única, individual, de significados, un modelo de mundo intercultural: plástico, permeable, mercurial - como de hecho se concibe la cultura desde el punto de vista antropológico-. Para usar una analogía, podríamos decir que una persona que desarrolla una personalidad intercultural tiene las habilidades de un camaleón: cambia de colores, de mirada, de perspectiva, según los interlocutores con los que se relaciona, 
sin dejar de ser la misma persona, planteamiento que hace eco de la definición de conciencia intercultural contenida en MRE, como ya lo hemos visto.

Gracias a la explicación del funcionamiento complejo de la competencia comunicativa de Cantero podemos concluir, recapitulando, que a mayor desarrollo de la competencia intercultural, es decir, de una identidad cultural no monolítica sino que permeable y en constante enriquecimiento cognitivo por el contacto con modelos de mundo diferentes, mayores posibilidades tendrá un hablante de activar exitosamente su competencia mediadora. Es una condición de posibilidad porque la interacción de ambas competencias depende del desarrollo de la competencia estratégica: la capacidad de aprovechar los recursos que se posean y generar nuevos para afrontar las dificultades que surjan en encuentros comunicativos inéditos a la experiencia habitual.

Con base en lo anterior, en términos ideales describiríamos a un mediador eficaz como la persona con una competencia estratégica e interactiva adecuadamente desarrolladas puesto que sabe conjugar hábilmente su competencia intercultural y mediadora para realizar acciones de mediación específicas, al poner en práctica microhabilidades lingüísticas como traducir, interpretar, parafrasear, intermediar, adecuar, sintetizar, apostillar y citar (Cantero y De Arriba 2004). Cabe añadir con énfasis las habilidades de negociar y conciliar, para aproximarnos a un sentido de competencia intercultural más afín con las capacidades que se requiere formar con el que afrontar los retos del desarrollo social y político en situaciones de conflicto por intolerancia cultural o inequidad.

Por ello, a continuación queremos seguir problematizando la noción de competencia intercultural que viene del contexto europeo para llegar a plantear una que sea coherente con estas circunstancias de naturaleza política en el contexto latinoamericano, y para insertarla en el modelo de competencia comunicativa de Cantero, indicando su desglose en tres dimensiones de aprendizaje: conocimientos, actitudes, habilidades o destrezas, que faciliten el diseño de intervenciones didácticas. 


\section{UNA NOCIÓN DE COMPETENCIA INTERCULTURAL PARA LOS CONTEXTOS EDUCA- TIVOS LATINOAMERICANOS}

Al pasar al terreno de las discusiones teóricas sobre cómo definir la interculturalidad en Latinoamérica, nos parece que los planteamientos no difieren en su totalidad con la perspectiva europea, porque ambos discursos coinciden en el objetivo sociopolítico de favorecer la convivencia no conflictiva de grupos culturales diferentes en función de proyectos orientados al desarrollo social y prosperidad general. Por ejemplo, perfectamente se podría adoptar para nuestro contexto la declaración de propósitos que el Consejo de Europa establece en relación con el desarrollo de una competencia comunicativa, plurilingüe y pluricultural en los europeos: «mejorar la calidad de la comunicación entre los [latinoamericanos] que usan distintas lenguas y tienen distintos orígenes culturales» porque «la mejora de la comunicación produce una mayor movilidad y un contacto más directo, lo que a su vez provoca una mayor comprensión y una colaboración más intensa», y porque también interesa promover «los métodos de aprendizaje y enseñanza que ayudan tanto a los estudiantes jóvenes como a los adultos a desarrollar las actitudes, los conocimientos y las destrezas necesarias para llegar a ser más independientes a la hora de pensar y actuar y, a la vez, más responsables y participativos en relación con otras personas» y porque así «el trabajo contribuye a fomentar la ciudadanía democrática» (2001: XI-XII). Sin duda, todo sistema educativo proclama esta meta y es la promesa por cumplir más ansiada para sectores de la sociedad civil tradicionalmente marginados.

Tomando como punto de partida una propuesta específica, al comparar los rasgos que Duarte (1999) enumera para describir el tipo de sociedad intercultural a la que aspiran en la sociedad guatemalteca, se puede notar similitud con los planteamientos del discurso europeo:

- El reconocimiento del otro como interlocutor en igualdad

- Conocer las otras culturas distintas a la propia

- Valorar la diversidad cultural en los lindes de la nación

- Superar la desconfianza, temores, prejuicios, estereotipos

- Aspirar a un aprendizaje mutuo, con la voluntad para hacerlo

- Procurar una comunicación efectiva 
En esta propuesta se añade que las relaciones interculturales deben caracterizarse por el intercambio, la convivencia, el interés positivo por el otro, la confianza, la cooperación, la regulación pacífica del conflicto, el diálogo y el debate. Cabe añadir, según las ideas del filósofo cubano Fornet-Betancour (2001), la voluntad de traducción recíproca, de compartir y trasformar, y de conjugar los modos de sentir para crear un nuevo lenguaje común.

Por supuesto que estas similitudes resultan superficiales al trasluz de un análisis contextualizado y considerando su génesis histórica; en otras palabras, los planteamientos difieren muchísimo por la estructura de poder desde la cual se enuncian. Por ejemplo, de postulados como «el reconocimiento del Otro como interlocutor en igualdad»o «la regulación pacífica del conflicto», podemos deducir que aluden a una situación de injusticia social que se desea revertir, cosa que obviamente en MRE no se menciona en absoluto. Que mediante el desarrollo de una personalidad intercultural en todos los miembros de un país se genere la tan deseada ciudadanía democrática es algo que está aún por verse en muchos países latinoamericanos, como ya lo decíamos antes, ya que la curiosidad positiva por el otro diferente no necesariamente irá acompañada de una vinculación social en un plano de igualdad. Garantizar correspondencia entre estas actitudes implica un trabajo de formación ciudadana que se cruza con las lógicas de las luchas que se dan al interior del campo político, como bien lo plantea Pierre Bourdieu en su teoría de los campos. Lo cierto es que mientras se pierda de vista el interés en y la importancia de que prevalezca el bien común, los agentes lucharán por ocupar las mejores posiciones en su propio beneficio individual o de su grupo social. Es el tipo de retos que un programa educativo intercultural en favor de sectores sociales marginados debe atender.

En otra comparación, si leemos las primeras reflexiones del porqué de la necesidad de aprender lenguas, la respuesta europea es simple: «People want to be able to make contact with each other as people, to exchange information and opinions, talk about experiences, likes and dislikes, to explore our similarities and differences, the unity in diversity of our complicated and crowded continent» (J. Trim, en van Ek 1993: VI). La 
respuesta cambia radicalmente si nos trasladamos al contexto latinoamericano: en la mayoría de los países, la necesidad de aprender otras lenguas por parte de la población indígena y afrodescendiente —entiéndase: aprender bien la lengua oficial- es un asunto de sobrevivencia, de cobrar visibilidad, de acceso a los derechos sociales. Mientras para el Consejo de Europa se trata de favorecer la movilidad y oportunidades de vida de sus ciudadanos, y quizá también la precaución de evitar conflictos con la población inmigrante, para el contexto latinoamericano se trata de gestar un cambio social más profundo, se trata de instaurar — de forma consensuada - un nuevo proyecto de Estado, que fortalezca la valoración de la diversidad cultural y mecanismos de participación incluyente, donde la interculturalidad signifique «poder compartido» (Moya 2005).

Esto significa que el Estado debería proveer de las condiciones legales e institucionales necesarias para que el aprendizaje de la competencia intercultural sea accesible a todos los miembros de la nación, y casi que obligatorio para aquellos que históricamente han sido los voceros de formas tradicionales de discriminación - racismo, xenofobia, machismo, etc.- . Es decir, la viabilidad, sostenibilidad y éxito de un programa alternativo de educación que atienda las demandas de miembros de la nación que han sido simbólica y estructuralmente marginados del campo de poder, pasa ineludiblemente por la aplicación de cambios de igual índole que resuelvan problemas latentes de inequidad social y por un cambio de mentalidades.

De lo contrario, programas de educación intercultural bilingüe corren el riesgo de convertirse en paliativos, en soluciones superficiales que solamente postergan y evaden los pasos decisivos para ceder cuotas de poder por parte de la clase gobernante, al menos a través de mecanismos de participación que garanticen incidencia e inclusividad para todas y todos. De acuerdo con el análisis crítico de Sartorello (2011), esto puede identificarse como la «retorización» del concepto de interculturalidad, estrategia discursiva y política mediante la cual los discursos neoliberales han sabido apropiarse de este término y lo han convertido en el nuevo eslogan de políticas neoindigenistas que problematizan la diversidad cultural, pero con la agenda oculta de minimizar la 
discusión en torno a los problemas de raíz más acuciantes: las disimetrías socioeconómicas, el derecho al desarrollo equitativo, etcétera.

Por lo tanto, toda nuestra formulación teórica sobre la competencia comunicativa y la intercultural como núcleos de la enseñanza de lenguas se ubica inicial y evidentemente en un planteamiento desiderativo, que al momento de su puesta en práctica mediante un diseño curricular, un proyecto educativo de Centro, un plan de clase, pues sin duda encontrará dichas limitantes y las de su propia novedad, confrontada con esquemas o estructuras tradicionales tanto pedagógicas como en la gestión institucional procedimientos administrativos, disponibilidad de presupuesto para formación docente y materiales didácticos, legislaciones etnocéntricas-. Más grave aún, como bien lo señala Ruth Moya (2005), la dificultad de cambiar viejos modelos educativos, y la visión de mundo que los sostiene, tan arraigados en la manera de pensar de los maestros, puede ser uno de los obstáculos más duros de vencer. Desde la teoría de los estudios poscoloniales, el fenómeno se reconoce como la persistencia de una mentalidad colonizada que opone una resistencia pasiva, inconsciente, o incluso activa en contra de, por ejemplo, la revalorización de la propia cultura en relación con la cultura dominante.

Sin embargo, en la actualidad, precisamente la interculturalidad, en tanto paradigma educativo, ha demostrado su potencial como antídoto contra estas lógicas hegemónicas, ya que se puede potenciar en el sentido de «una alternativa al enfoque homogeneizante del sistema educativo tradicional occidental [porque persigue] una transformación de las relaciones sociales ante la diversidad desde la equidad, pertinencia y relevancia curricular; ser una herramienta para la construcción de una ciudadanía inclusiva en lugar de excluyente» (López 1997: 57). En tal sentido, el modelo sobre la competencia comunicativa y la noción de interculturalidad que hemos venido desarrollando a partir de las investigaciones en el campo de la enseñanza de lenguas en Europa, adquiere en nuestro contexto educativo tres usos instrumentales: como paradigma para crear una nueva pedagogía de la diversidad, como metodología para una revisión y renovación del currículo escolar (ibíd: 58), y como plataforma crítica desde la cual denunciar «la sordera endémica de las etnocracias», que han sabido mantener las condiciones de gobernabilidad a favor de sus intereses (Moya 2005: 12). 
Creemos que la burla a las esperanzadas expectativas de los sectores marginados de la sociedad civil que tanto añoran ver cumplida la promesa del desarrollo equitativo, de una educación que realmente los conduzca a mejores condiciones de vida, puede confrontarse mediante la educación intercultural como una estrategia al alcance de los educadores: apostar por la enseñanza de la competencia intercultural en el marco amplio de la enseñanza de la competencia comunicativa, sobre todo en los espacios educativos propios de los grupos sociales que alimentan posturas negativas hacia la diferencia cultural y lingüística.

Luego de insistir en que la definición de competencia comunicativa e intercultural dentro de las coordenadas de la educación en Latinoamérica debe tomar en cuenta estos matices de naturaleza política, no hay por qué desaprovechar las importantes innovaciones de los modelos teóricos revisados. De MRE:

- El enfoque basado en la acción y la noción de agente social.

- La diferenciación entre competencias generales y específicas.

- La definición de conciencia intercultural como componente de las competencias generales, y sus indicadores como conocimiento declarativo y procedimental en dichas competencias.

Del modelo de Cantero:

- Las categorías de competencias generales - lingüística, discursiva, cultural y estratégica - y de las competencias específicas — producción, recepción, interacción y mediación.

- Los esquemas de funcionamiento complejo: el triángulo múltiple y la estrella comunicativa.

- $\quad$ La definición de competencia intercultural.

Derivado de todo lo anterior, y en función de la formación que pensamos se requiere para afrontar los retos ya mencionados, proponemos una definición de competencia comunicativa - provisional, adaptable y perfectible, según cada contexto de aplicación - como el conjunto de conocimientos, habilidades y destrezas que una 
persona adquiere, y puede desarrollar sin punto final, para interactuar con éxito como agente social que es con los demás miembros de su nicho social, así como los de cualquier otro ámbito cultural con los que entre en contacto, de manera que sea capaz de enfrentar situaciones de interacción inéditas en su experiencia, ya sea de tipo conflictivo o de simple amable intercambio social, todo lo cual debe redundar positivamente en su desarrollo como comunicador, discente, agente social y persona, y a su vez favorecer el desarrollo de su comunidad sociopolítica hacia condiciones de prosperidad equitativa general.

Ese conjunto de conjunto de conocimientos, habilidades y destrezas de la competencia comunicativa se organiza en competencias generales -lingüísticas, discursivas, estratégicas, culturales - y acciones comunicativas específicas —producción, recepción, interacción y mediación-, siendo la relación entre la competencia estratégica - el saber aprender o zona de desarrollo próximo- y la competencia interaccional motor de la competencia comunicativa, lo que puede dar como producto la trasformación de la competencia cultural en una competencia intercultural, que condiciona a su vez el funcionamiento idóneo de la competencia mediadora, de acuerdo con el funcionamiento complejo que ilustra la estrella comunicativa.

Dicha competencia intercultural constituye un conjunto específico de disposiciones y aptitudes necesarias para descubrir la otredad y gestionar adecuadamente una relación con ésta:

- $\quad$ el conocimiento, la percepción y la comprensión de la diversidad cultural;

- $\quad$ aceptación de la diferencia como la norma en lugar de una homogeneidad imaginaria;

- conciencia de la relación entre el modelo de mundo propio y los otros modelos de mundo - contrastes y puntos en común;

- toma de conciencia del modo en que una cultura puede desfigurarse en estereotipos desde miradas externas;

- capacidad de relativizar los parámetros de la propia cultura — valores, creencias, prácticas; 
- capacidad de aprendizaje constante y flexibilidad para el enriquecimiento del modelo de mundo;

- capacidad de desarrollar actitudes de respeto, tolerancia, confianza, cooperación, y

- capacidad de desarrollar habilidades de aprendizaje y mediación lingüística, en especial para la negociación y conciliación.

Todo lo anterior se puede sistematizar en tres dimensiones de aprendizaje: conocimientos, actitudes y habilidades o destrezas, como efectivamente lo propone Teresa Aguado (2003), con el importante aporte de desglosar los indicadores específicos de cada dimensión, y relacionarlos coherentemente: cada indicador de la primera dimensión tiene su correlato en las dos dimensiones siguientes. En otras palabras: el aprendizaje de unos conocimientos debe ir acompañado del aprendizaje de unas habilidades y unas aptitudes consecuentes. Esta sistematización de los indicadores de la competencia intercultural resulta muy ventajosa al momento de diseñar las intervenciones didácticas y operativizar así la formación que se busca lograr en un programa educativo intercultural. Sin duda, enriquece la breve lista de microhabilidades lingüísticas que Cantero había identificado para la competencia intercultural y de mediación.

\begin{tabular}{|c|c|}
\hline \multicolumn{2}{|r|}{ Dimensiones de la Competencia Intercultural } \\
\hline \multirow{6}{*}{ 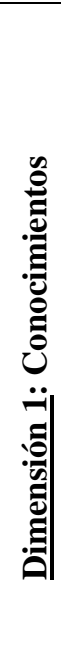 } & $\begin{array}{l}\text { 1.1 Conocimientos sobre la propia cultura: historia, creencias (religiosas, morales...), valores, } \\
\text { características (vestido, comida, territorio...), productos culturales (artesanía, mitología...), } \\
\text { procesos generales de interacción social }\end{array}$ \\
\hline & 1.2 Conocimientos sobre otras culturas de contacto: ídem todo lo anterior \\
\hline & 1.3 Comprensión de la diversidad cultural \\
\hline & $\begin{array}{l}\text { 1.4 Toma de conciencia del modo en que una cultura puede desfigurarse en estereotipos desde } \\
\text { miradas externas, y cómo influyen en la relación con dicha cultura, es decir, cómo se elaboran y } \\
\text { mantienen los estereotipos y cómo nos influyen para actuar }\end{array}$ \\
\hline & 1.5 Conciencia del condicionamiento cultural de todo comportamiento \\
\hline & $\begin{array}{l}\text { 1.6 Comprensión del significado y consecuencias en la conducta de: sexismo, racismo, } \\
\text { xenofobia, prejuicios y todo tipo de discriminación por la diferencia }\end{array}$ \\
\hline
\end{tabular}




\begin{tabular}{|c|c|}
\hline \multirow{9}{*}{ 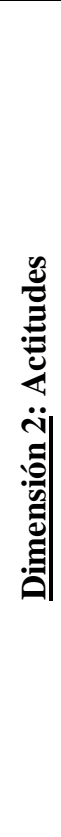 } & $\begin{array}{l}\text { 2.1 Curiosidad, interés positivo y apertura hacia el otro, es decir, capacidad de aprendizaje } \\
\text { constante y flexibilidad para el enriquecimiento del modelo de mundo }\end{array}$ \\
\hline & 2.2 Aceptación de la diferencia como la norma en lugar de una homogeneidad imaginaria \\
\hline & $\begin{array}{l}\text { 2.3 Aceptar una relación horizontal con la diferencia cultural, manifestando confianza en el } \\
\text { valor de su propia cultura }\end{array}$ \\
\hline & $\begin{array}{l}\text { 2.4 Capacidad de reconocer contrastes y puntos en común con otras culturas, es decir, aceptar la } \\
\text { validez de otras maneras de pensar }\end{array}$ \\
\hline & $\begin{array}{l}\text { 2.5 Capacidad de relativizar los parámetros de la propia cultura, es decir, ser autocríticos y así } \\
\text { incrementar la comprensión de la propia cultura }\end{array}$ \\
\hline & 2.6 Capacidad de ponerse en los zapatos del otro \\
\hline & 2.7 Demostrar respeto y tolerancia \\
\hline & 2.8 Disponibilidad a la cooperación, negociación y conciliación \\
\hline & $\begin{array}{l}\text { 2.9 Rechazo a manifestaciones de racismo, sexismo, xenofobia, prejuicios y discriminaciones, } \\
\text { en general }\end{array}$ \\
\hline \multirow{8}{*}{ 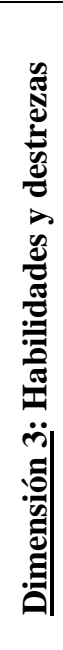 } & $\begin{array}{l}\text { 3.1 Aprendizaje, habilidad de buscar y adquirir nuevos conocimientos acerca de otras culturas } \\
\text { de contacto. Saber cómo preguntar a personas de otras culturas sobre sus costumbres }\end{array}$ \\
\hline & 3.2 Interpretación y comprensión de los nuevos conocimientos sobre otras culturas. \\
\hline & 3.3 Mediación lingüística: escuchar, negociar, dialogar, resolución de conflictos \\
\hline & $\begin{array}{l}\text { 3.4 Analizar y reconocer pautas comunicativas y conductas que conllevan manifestaciones de } \\
\text { racismo, sexismo, xenofobia, prejuicios y discriminaciones en general }\end{array}$ \\
\hline & 3.5 Comparación de formas culturales diferentes, o entre la cultura propia y otras \\
\hline & 3.6 Comprensión de las diferencias, del origen de los malentendidos \\
\hline & $\begin{array}{l}\text { 3.7 Interacción: habilidad de utilizar todos estos conocimientos en situación reales de } \\
\text { interacción comunicativa }\end{array}$ \\
\hline & 3.8 Confianza y tranquilidad para abordar temas conflictivos \\
\hline
\end{tabular}

Como lo hemos anunciado, en este punto queremos esbozar, dentro de las posibilidades de este espacio, nuestra propuesta curricular para implementar la enseñanza de la competencia intercultural. Tomamos como punto de partida el potencial que van Ek identifica en los textos literarios para el aprendizaje de la competencia sociocultural. En nuestras palabras: partimos de la certeza de que las narraciones juegan un papel protagónico en la trasmisión de significados culturales y por ende en la formación identitaria de las personas mediante la educación en el hogar, puesto que inciden en la maduración sicológica y configuración de la personalidad de los infantes, cuando permiten un contacto íntimo y personal con personajes, comportamientos, formas de pensar, eventos tradicionales, rituales, etcétera, en el plano de la imaginación. 
Por tanto, nuestra certeza en este potencial formativo de las narraciones determina los rasgos metodológicos de nuestra propuesta curricular. El objetivo es propiciar la modelación de la conciencia de los participantes en el proceso educativo — sean niñas y niños o docentes en formación- en función de los indicadores de la competencia intercultural, los cuales reflejan a su vez los principios del paradigma intercultural. Se trata de trabajar la identidad, no la normativa gramatical de un código lingüístico, no se trata de una propuesta orientada a la promoción de la lectura, el aprendizaje de la lectoescritura, el aprendizaje de segundas lenguas o la educación literaria, sino que primordialmente al aprendizaje de un comportamiento específico, de una manera de ser: sustentados por la competencia intercultural. Lo cual no significa que esos otros aprendizajes se menoscaben, sin embargo no cumplen la función detonadora de la acción pedagógica.

Proponemos como recurso didáctico nuclear un Canon Formativo Intercultural, compuesto por narraciones — sean cuentos, leyendas, crónicas, etc.- que tome en cuenta la tradición oral de los pueblos originarios en el contexto social donde se realice la intervención didáctica, y dando cabida también a muestras de tradición oral universal, manejando como criterio de selección la presencia de elementos comunes en el contexto de personajes o temas. Es decir, se debe buscar crear en el sentido de la antología literaria un encuentro de culturas con base en los mínimos comunes.

El soporte para el Canon Formativo Intercultural es el formato del libro-mudo: ilustraciones sin texto, que muestran directamente el suceder de las acciones de una historia, y que tendrían su complemento textual en la narración oral del docente, quien estaría emulando al cuentacuentos de la tradición oral local. De esta forma, el cuento o leyenda o crónica o... queda desvinculado de su formato lingüístico, y expone sus contenidos culturales directamente a través de la imagen, solamente con el apoyo en una narración oral, formato original de la Literatura de Tradición Oral. Así, dado que la plataforma gráfica será la misma para el alumnado meta - que puede observar un perfil multicultural-, no importa la lengua en que se trasmita el relato, que sirva como 
vehículo de comunicación, lo importante es cómo se cuenta, cómo se comenta y qué actividades se realizan a partir de éste para generar los aprendizajes deseados.

Sugerimos que se realicen actividades mediante las cuales niñas y niños se hagan dueños de las narraciones y así interioricen los significados culturales en juego, por ejemplo mediante modificaciones de algunos elementos - personajes, título, final, soluciones a conflictos, etc.--, para que inventen, que sientan la historia como un objeto maleable, propio, que la vivan emotivamente como algo personal, que se impliquen, que queden inmersos en la dimensión ficticia de la narración como vehículo del espíritu, de los sentimientos. En fin, queremos que se potencie la imaginación y la creatividad a partir de los conocimientos culturales en función de relaciones interculturales.

Evidentemente, esta propuesta didáctica trabaja a nivel simbólico para conectar con los significados más profundos de las narraciones, que constituyen los elementos comunes identificados previamente durante la selección de textos literarios, aquéllos conforman el Canon Formativo Intercultural. Creemos que de esta manera se podrán generar conexiones entre los miembros del espacio social, quizá tradicionalmente confrontados o desvinculados. Valiéndonos de las palabras de Ruiz de Lobera, esperamos que el Canon Formativo Intercultural sea una «puerta [para] la penetración reflexiva en $m i$ sistema de valores a través de la comprensión de $t u$ sistema de valores» (2004: 40).

Finalmente, resumimos en sus pasos generales la secuencia metodológica que hemos diseñado para el trabajo de aula:

1. Se debe empezar con una actividad de animación que puede ser un juego o la confección de una manualidad, o ambos, u otro tipo de actividad lúdica.

2. La lectura visual del libro-mudo con apoyo de la narración oral, incluye indicaciones para la observación y disfrute de las imágenes.

3. Ejercicios de predicción o anticipación a partir de la portada, por cada ilustración, sobre el final o el futuro — qué pasará después.

4. Verificación de la comprensión lectora, aclaración de dudas y explicación de conceptos clave. 
5. Ejercicios de análisis y/o reflexión en función de los indicadores de la competencia intercultural, para ser trabajados en su aprendizaje y desarrollo por parte de los discentes. Por ejemplo: inducción a la empatía, ponerse en los zapatos del otro, conectar con la experiencia propia, ejercicios de comparación — reconocimiento de diferencias y similitudes.

6. Realización de una actividad plástica, es decir, crear un objeto simbólico que quede vinculado a los aprendizajes, a las ideas compartidas, a la memoria de las experiencias con el libro-mudo. Esto es crucial sobre todo en el caso de trabajar con relatos de una etnia diferente a la propia, de manera que ese otro universo cultural recién conocido - al cual niñas y niños o docentes en formación fueron trasladados - se pueda sentir cercano por efecto del contacto con un objeto lúdico concreto.

7. Ejercicios de creación y manipulación literaria: crear, cambiar o mezclar el título, el inicio, el final, los personajes, los lugares, la época, etcétera.

8. Realizar actividades alternativas y/o complementarias, como la práctica de escritura en L1 y/o L2, dramatizaciones, investigaciones, manualidades, teatro con títeres...

Se debe procurar que la mayoría de actividades conlleve la práctica de las cuatro habilidades específicas de la competencia comunicativa: percepción, producción, mediación y estrategia, así como la práctica de microhabilidades orales de mediación: resumir y sintetizar, parafrasear, apostillar, intermediar, interpretar, negociar.

Los términos ideales y propositivos de nuestro planteamiento quizá no nos dejan ver de momento las limitantes probables para que la implementación de estos lineamientos curriculares sea exitosa en el ámbito escolar o en el de la formación docente; creemos sin embargo que estas provendrán más bien de factores externos, si no se dan condiciones de apoyo legal e institucional en el sistema educativo, y si no se dan las condiciones para la existencia y el uso en el espacio público de las lenguas y culturas discriminadas simbólicamente. 
Por lo anterior, queremos enfatizar una vez más que este desarrollo de la competencia comunicativa-intercultural debe mantener como norte un objetivo extralingüístico clave: que las personas como agentes sociales puedan participar activamente desde su campo de acción individual en la construcción de una sociedad respetuosa de la diversidad, donde se fortalezcan los canales de cooperación y la confianza, la igualdad de derechos y oportunidades, el interés positivo por el otro, la voluntad de aprendizaje y traducción recíproca, de compartir y transformar, de conjugar los modos de sentir para que se produzca de la manera menos conflictiva posible, con seguridad y optimismo, una transculturación positiva hacia un proyecto de Estado nación intercultural y plurilingüe, lo cual implica abandonar la intención de proteger identidades esenciales como si fueran inmunes al cambio histórico, a la evolución cultural, dando prioridad a la sobrevivencia y bienestar de las personas, quienes se relacionan interculturalmente gracias a la existencia estable de una ciudadanía democrática y al respeto de los derechos humanos. 


\section{BIBLIOGRAFÍA}

Aguado, Teresa, 2003, Pedagogía Intercultural, McGraw-Hill, Madrid.

Canale, M. \& M. Swain, 1980, «Theoretical Basis of Communicative Approaches to Second Language Teaching and Testing», Applied Linguistics, n. 1, pp. 1-47.

Consejo de Europa, 2001, «Marco Común Europeo de Referencia para las Lenguas: Aprendizaje, Enseñanza, Evaluación», en http://cvc.cervantes.es/obref/marco [consulta: enero de 2008], Instituto Cervantes.

Cantero, Francisco José, 2009, «Complejidad y competencia comunicativa», Congreso Horizontes de Lingüística Aplicada, Brasilia, Brasil.

Cantero, Francisco José y Antonio Mendoza, 2003, «Conceptos básicos en didáctica de la lengua y la literatura», en Didáctica de la Lengua y la Literatura, compilado por Antonio Mendoza, Pearson Educación, Madrid, pp. 33-78.

Cantero, Francisco José y Clara De Arriba, 2004, «Actividades de mediación lingüística para la clase de ELE», en http://www.mec.es/redele/revista2/cantero_arriba.shtml [consulta: marzo de 2008], Red ELE 2.

Duarte Méndez, Sergio C. (coord.), 1999, «Guía Sobre Interculturalidad. Primera Parte. Fundamentos Conceptuales», Proyecto Q’Anil, PNUD, Guatemala.

Fábregas Puig, Andrés, 2011, «La Relación entre los Pueblos Originarios y los Estados Nacionales de América Latina». Ponencia presentada en el IV Congreso Centroamericano de Antropología, Tegucigalpa, Honduras.

Fornet-Betancour, Raúl, 2001, «Lo intercultural: el problema de su definición», en http://www.aulaintercultural.org/IMG/pdf/betancour.pdf [consulta: septiembre de 2007], Aula intecultural. 
López, Luis Enrique, 1997, «La Diversidad Étnica, Cultural y Lingüística Latinoamericana y los Recursos Humanos que la Educación Requiere», Revista Iberoamericana de Educación n. 13, pp. 47-98.

Llobera, Miquel (ed.), 1995, Competencia comunicativa: documentos básicos en la enseñanza de lenguas extranjeras, Edelsa, Madrid.

Mendoza, Antonio y Francisco Cantero, 2003, «Didáctica de la Lengua y de la Literatura: Aspectos Epistemológicos», en Didáctica de la Lengua y la Literatura, compilado por Antonio Mendoza, Pearson Educación, Madrid, pp. 3-31.

Moya, Ruth, 2005, «Formación de Maestros e Interculturalidad», en Formación Docente y Educación Intercultural Bilingüe en América Latina (CD-ROM), compilado por Nicole Nucinkis y Luis Enrique López, Ministerio de Educación de Bolivia, Cooperación Técnica Alemana, GTZ, Bolivia.

Oliveras Vilaseca, Àngels, 1998, «Hacia la competencia intercultural en el aprendizaje de una lengua extranjera: estudio del choque cultural y los malentendidos», Tesis, Universitat de Barcelona, Barcelona.

Ruiz de Lobera, Mariana, 2004, Metodología para la formación en educación intercultural, Catarata, Ministerio de Educación, Cultura y Deporte, Madrid.

Sartorello, Claudio Stefano, 2011, «La interculturalidad entre Retórica y Crítica», Ponencia presentada en el IV Congreso Centroamericano de Antropología, Tegucigalpa, Honduras.

Trim, John (ed.), 1997, Language learning for European citizenship: final report of the Project Group (1989-96), Council of Europe Publishing, Council for Cultural Cooperation, Education Committee, Strasbourg. 
van Ek, J. A., 1993, Objectives for foreign language learning, Council of Europe, Strasbourg.

van Ek, J. A. y John Trim, 1991, Threshold Level 1990, Council of Europe Press, Council for Cultural Co-operation, Strasbourg.

Fecha de recepción: 20 de junio de 2010.

Fecha de aceptación: 29 de abril de 2011. 